\title{
2D material-enabled nanomechanical bolometer
}

\author{
Shu-Yuan Chiang, $†$ Yueh-Yuan Li, ${ }^{\dagger}$ Tien-Lin Shen, ${ }^{\dagger}$ Mario Hofmann, ${ }^{*}, \dagger$ Yang-Fang \\ Chen $^{*},+$ \\ ${ }^{\dagger}$ Department of Physics, National Taiwan University, Taipei, Taiwan
}

\section{Supporting Information.}

\section{Details on finite element simulation}

The heat transfer inside a PDMS slab was simulated using a finite element approach to numerically solve the heat diffusion equation. The thermal conductivity of PDMS was assumed to be $0.16 \mathrm{~W} \mathrm{~m}^{-1} \mathrm{~K}^{-1}$. The top side of a $3 \times 10 \mathrm{~mm}$ PDMS slab was irradiated with a laser of $1 \mathrm{~mm}$ spot size and $500 \mathrm{~mW}$ intensity. The heat flux was calculated considering the emissivity of graphene to account for absorption in this top layer. 


\section{Characterization of $\mathrm{MoS}_{\mathrm{s}}$}

The transmittance spectrum of the shear-exfoliated $\mathrm{MoS}_{2}$ nanosheets in Figure $\mathrm{S} 1$ shows the semiconducting property that the shorter the wavelength is, the more light it absorbs.

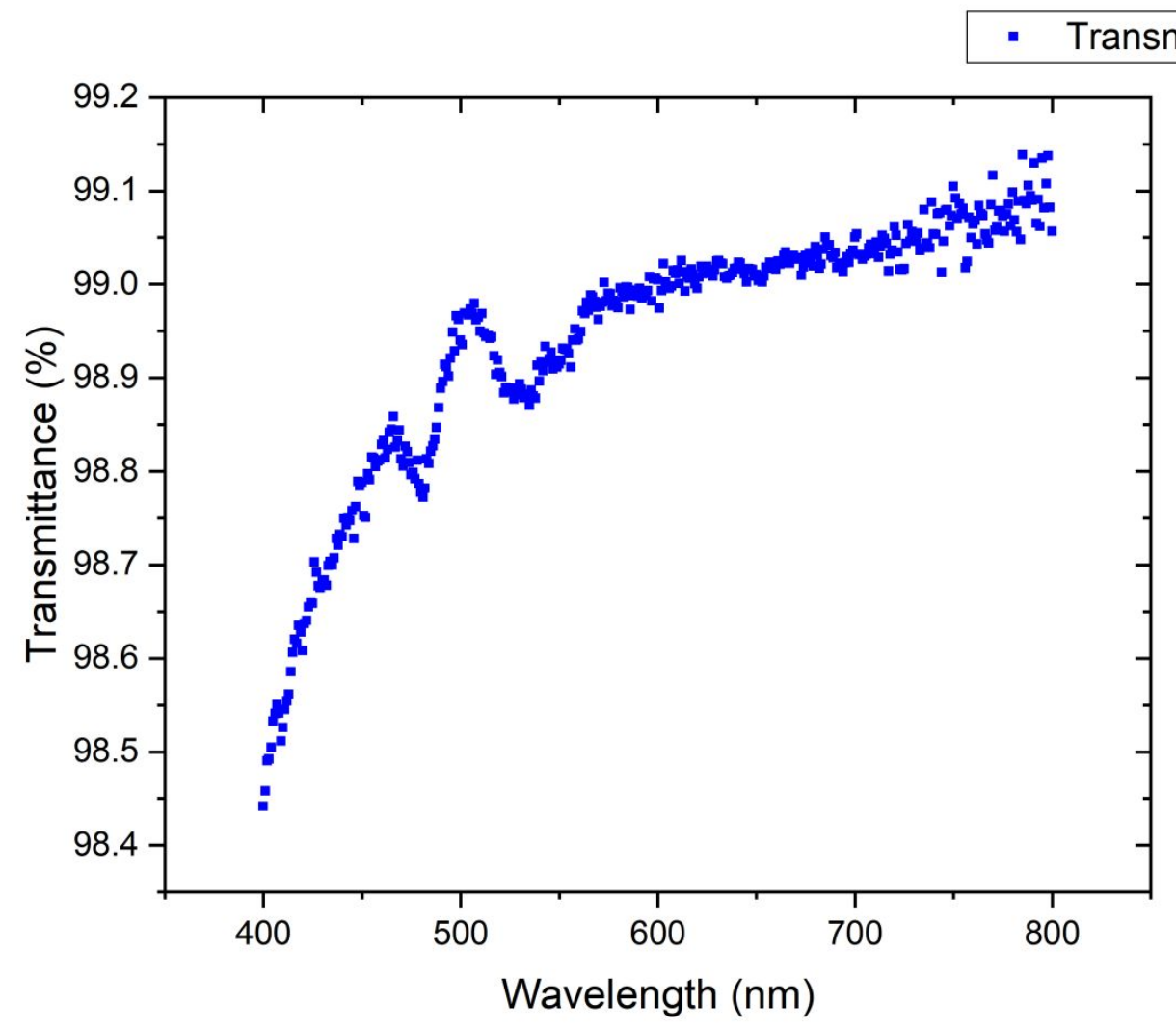

Figure S1. The transmittance spectrum of the $\mathrm{MoS}_{2}$ nanosheets employed.

Detailed characterization of the Raman spectra was conducted to study the presence of residue. Because of the overlap of graphene Raman features with the vibrational bands of the solvents, we investigate $\mathrm{MoS}_{2}$ Raman spectra and focus on NMP residue, since the 
solvent has a higher boiling point than IPA and is thus expected to be harder to remove.

We do not observe discernible peaks in any of the regions where NMP bands should occur, ${ }^{1}$ indicating the absence of significant residue.

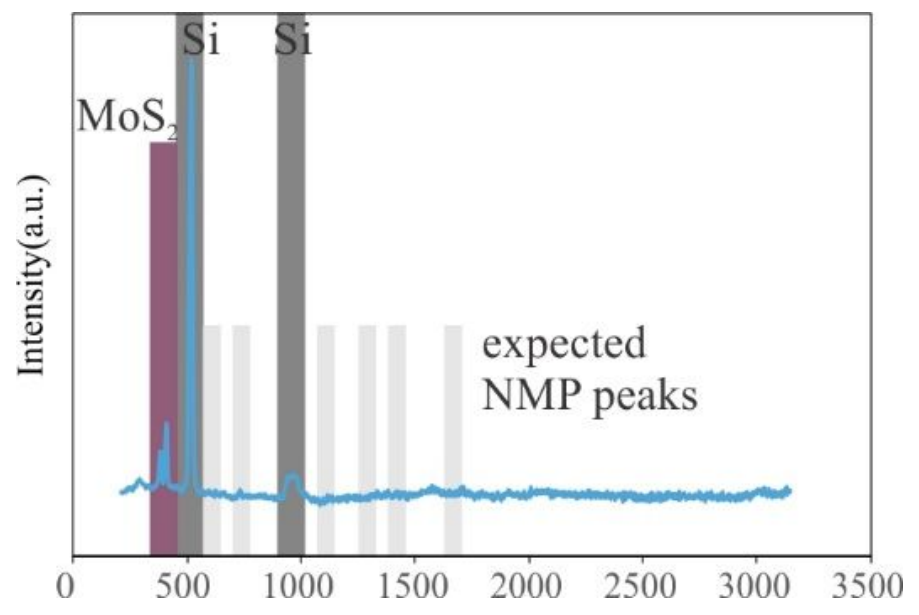

Figure S2. Extended-range Raman spectrum of $\mathrm{MoS}_{2}$ flakes deposited from NMP solution onto $\mathrm{Si} / \mathrm{SiO}_{2}$ wafer

Closer inspection of the $\mathrm{MoS}_{2}$ peaks in Figure 1d reveals a distance between $\mathrm{A}_{1 \mathrm{~g}}$ and $\mathrm{E}_{2 \mathrm{~g}}$ peaks $\left(24 \mathrm{~cm}^{-1}\right)$ permits the extrapolation towards the average flake thickness to values of approximately 5 layers ${ }^{2}$. This low thickness is in agreement with the low absorption of thin flake films (Figure S1). 


\section{Fabrication and characterization of edge-on irradiated bolometer devices}

The edge-on device was fabricated by drop coating. After dropped at the end of the cut, the solution automatically flowed through and filled the cut due to the capillary action. This phenomenon is useful in such full-solution process which significantly reduces the fabrication cost.

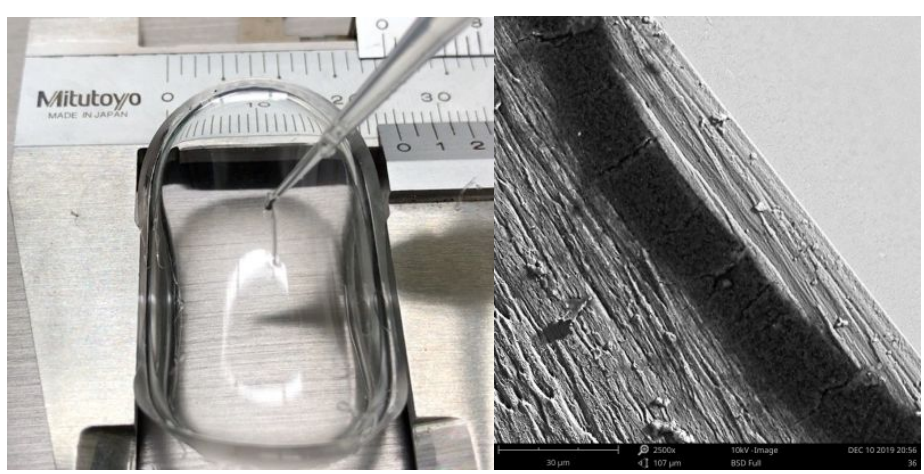

Figure S3. (Left) photograph of drop-coating process into PDMS gap, (right) crosssectional SEM image of $\mathrm{MoS}_{2}$ film formed in PDMS gap. 


\section{Details on Gauge factor}

We strain the $\mathrm{MoS}_{2}$ device with a manual stage micrometer and measure the resistance.

The result (Figure S3) shows the resistance-strain relationship that resistance increases

nonlinearly with strain. According to the definition of gauge factor (GF):

$$
G F=\frac{\Delta R / R}{\varepsilon}
$$

,where $R$ and $\varepsilon$ each mean resistance and mechanical strain, the gauge factor is up to 2.2

$\times 10^{3}$.

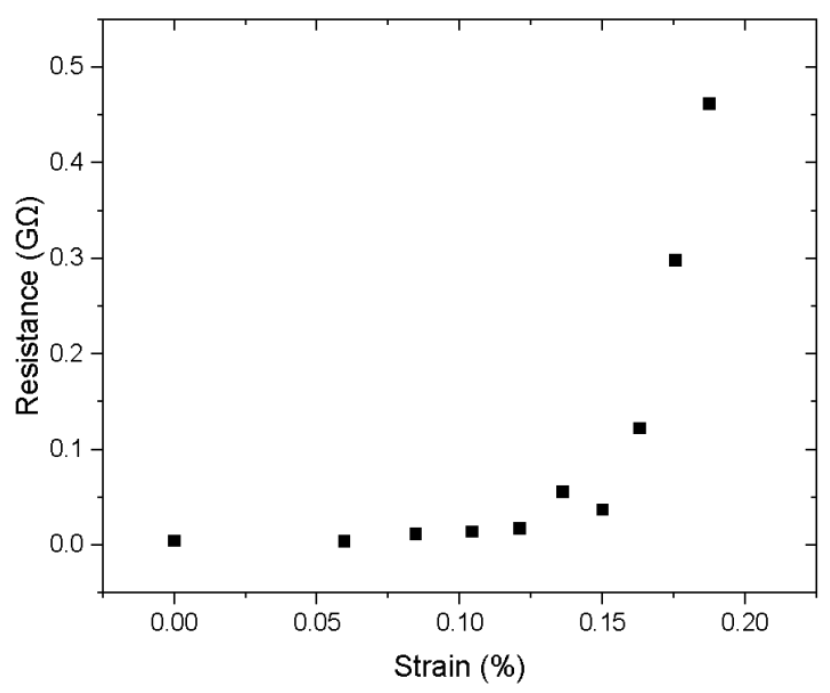

Figure S4. The relationship between the resistance of $\mathrm{MoS}_{2}$ bolometer and strain. 


\section{Temperature evolution of PDMS}

Finite element simulation was employed to characterize the time evolution of the temperature in our sample and explain the observed asymmetric response in Figure 4c. In the simulation the sample was irradiated with a laser for $1 \mathrm{~s}$ and a sharp increase in temperature was observed (Figure S4). Cooling does not proceed as efficiently since it depends on the thermal conduction through the PDMS. We therefore observe that a much longer time is required to reach the original temperature. These results are in agreement with the sensors response in Figure 4c. Under repeating irradiation under high duty cycles, the material does not have sufficient time to reach room temperature and instead an increase in average temperature is predicted. This effect can be seen in the slowly varying component of the photocurrent in Figure 3c.

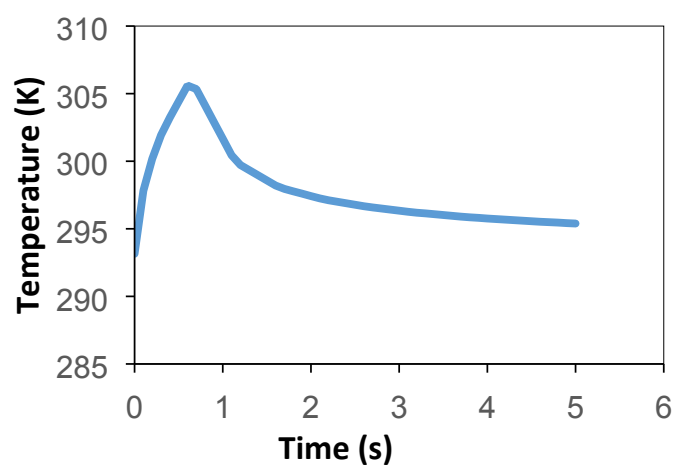


Figure S5. Temperature of PDMS surface after laser irradiation between $0 \mathrm{~s}$ and $1 \mathrm{~s}$. 


\section{Noise-equivalent power}

Noise-equivalent power (NEP) is a factor for sensitivity defined by the incident laser power which generates a signal-to-noise ratio of 1 per unit bandwidth of noise. To calculate the NEP from the current response of the thermal effect (Figure 4c), the spectrum of the noise is generated by FFT (Figure S5). Using the noise spectrum, the room-mean-square (RMS) of the noise portion of the spectrum per unit bandwidth of $1.11 \times 10^{-11} \mathrm{~A} \mathrm{~Hz}^{-1 / 2}$ is extracted, which is set as identical to the laser-generated signal to obtain the NEP. From the tendency in Figure $4 \mathrm{c}$, the laser power can be extracted as $5.67 \times 10^{-12} \mathrm{~W} \mathrm{~Hz}^{-1 / 2}$ as NEP.

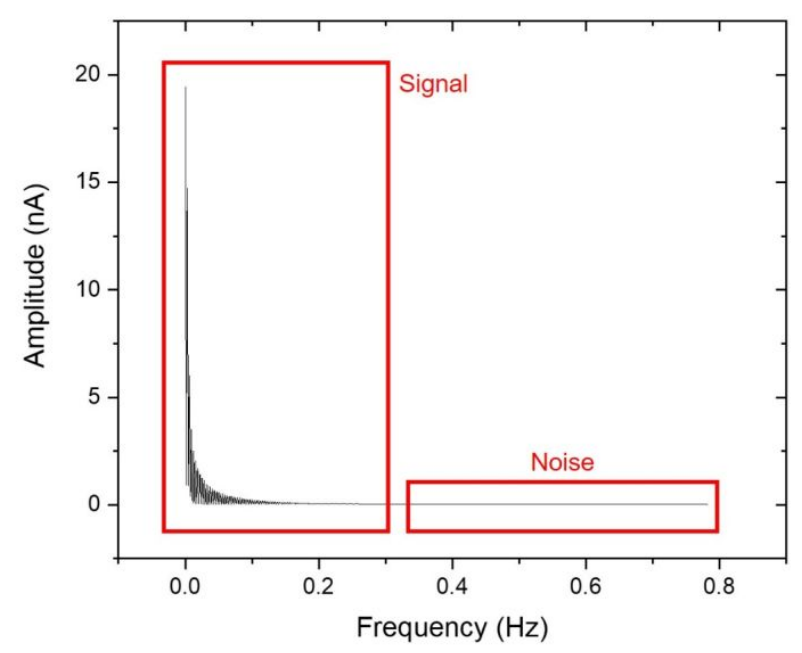

Figure S6. The spectrum of photoresponse of thermal effect shown in Figure 4c. 


\section{Laser spot size and spatial resolution}

The spot size of $808 \mathrm{~nm}$ laser is analyzed from the Figure S5 with software "ImageJ". To analyze the laser's spot size with the software, the image should be transformed into gray scale first. By choosing the range of the color level in gray scale, the laser's spot can be distinguished from the background as a color block. As a result, the spot size $3.10 \times 10^{5}$ $\mu \mathrm{m}^{2}$ (that is, $314.13 \mu \mathrm{m}$ as radius) can be obtained. The diameter of the laser spot shown in Figure S5 is equal to six times standard deviation of Gaussian distribution (99.8\% of the whole); thus, the standard deviation of the spot is $104.7 \mu \mathrm{m}$. The distribution of location dependence resistance (Figure 3f) can be fitted as a Gaussian very well, so it could be considered as a Gaussian distribution with standard deviation $104.5 \mu \mathrm{m}$. Besides, it is reasonable to assume the sensing distribution is also Gaussian-like. Therefore, it is a model base on the convolution of two Gaussian distributions. If

$$
f(x)=\frac{1}{\sqrt{2 \pi} \sigma_{f}} e^{-\frac{\left(x-\mu_{f}\right)^{2}}{2 \sigma_{f}^{2}}}
$$

and 


$$
g(x)=\frac{1}{\sqrt{2 \pi} \sigma_{g}} e^{-\frac{\left(x-\mu_{g}\right)^{2}}{2 \sigma_{g}^{2}}}
$$

, the convolution of two functions will be:

$$
h(x)=f(x) * g(x)=\frac{1}{\sqrt{2 \pi\left(\sigma_{f}^{2}+\sigma_{g}^{2}\right)}} e^{-\frac{\left(x-\left(\mu_{f}+\mu_{g}\right)\right)^{2}}{2\left(\sigma_{f}^{2}+\sigma_{g}{ }^{2}\right)}}
$$

Set $f(x)$ as the sensing distribution we trying to figure out, $g(x)$ as the Gaussian distribution of laser beam, and $h(x)$ as the location dependence resistance. Then the standard deviation of sensing distribution $\left(\sigma_{f}\right)$ be easy to figure out with standard deviation of laser beam $\left(\sigma_{g}\right)$ and location-related resistance $\left(\sqrt{\sigma_{f}^{2}+\sigma_{g}^{2}}\right)$ which are already known. As a result, $6.6 \mu \mathrm{m}$ as $\sigma_{f}$ can be figure out. The spatial resolution is based on the full width at half maximum (FWHM), and the relationship between FWHM and standard deviation is:

$$
\mathrm{FWHM}=2 \sqrt{2 \ln 2} \sigma
$$

Therefore, the FWHM of thermal sensing distribution can be figured out as $15 \mu \mathrm{m}$. 


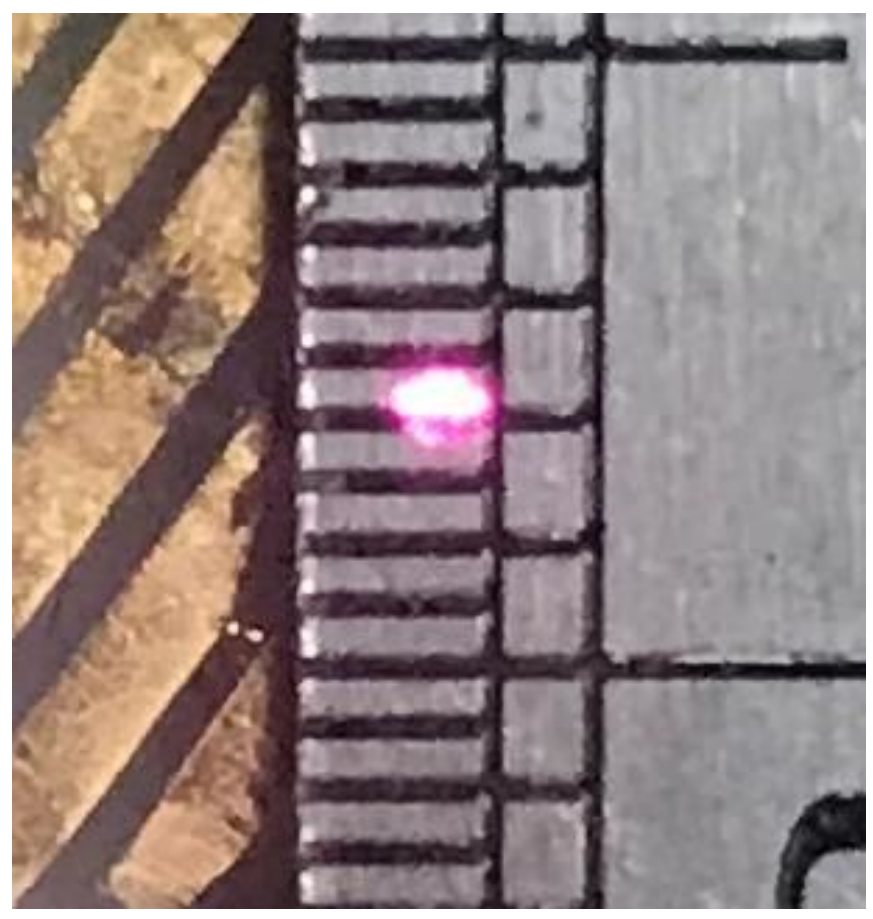

Figure S7. The picture of the laser spot. 


\section{REFERENCES}

(1) $\mathrm{Xu}, \mathrm{W} . ;$ Wang, H.; Tao, Y.; Zheng, X. The structural organization of N-methyl-2-pyrrolidinone in binary mixtures probed by Raman spectroscopy: Experimental and quantum chemical results. J. Raman Spectrosc. 2018, 49, 362-371.

(2) Ye, M.; Winslow, D.; Zhang, D.; Pandey, R.; Yap, Y. K. In Recent advancement on the optical properties of two-dimensional molybdenum disulfide (MoS2) thin films,

Photonics, 2015; Multidisciplinary Digital Publishing Institute: pp 288-307. 